\title{
生猪规模养殖粪污治理行为影响因素分析 基于意愿转化行为视角
}

\author{
赵俊伟 ${ }^{1,2}$, 姜 昊 ${ }^{1}$, 陈永福 ${ }^{2}$, 尹昌斌 $^{1}$ \\ (1. 中国农业科学院农业资源与农业区划研究所, 北京 100081; \\ 2. 中国农业大学经济管理学院,北京 100083)
}

\begin{abstract}
摘要: 为促进养殖户积极参与粪污治理, 基于意愿转化行为视角, 利用吉林、辽宁 2 省 25 县的 生猪规模养殖户调研数据, 通过对样本数据进行分组 (无意愿无行为、有意愿无行为、有意愿有 行为), 运用无序多分类 Logistic 回归模型从 “有无意愿” “有无行为” “意愿转化行为”三个维度 综合分析生猪规模养殖粪污治理行为的影响因素。结果显示: 养殖户的粪污治理意愿与治理 行为之间存在较大差异, 意愿与行为的不一致比率达 $26.8 \%$ 。影响养殖户粪污治理意愿转化行 为的因素主要与养殖规模、粪污消纳地面积、养殖净收益、周边群众與论、粪污处理技术、粪污 处理经济条件、粪污处理相关培训、政府补贴和政府监管等相关性较大, 并且对意愿向行为转 化具有显著的促进作用,而养殖年限成为养殖户粪污治理意愿转化行为的阻碍因素。
\end{abstract}

关键词：生猪规模养殖;粪污治理;治理行为;一致性;影响因素

统筹推进畜牧业发展和畜离粪污治理, 是贯彻绿色发展理念, 建设美丽中国的必然 要求。近年来, 随着畜禽规模化、集约化养殖进程加快，区域性粪污产生量越来越大 ${ }^{[1]}$, 据估计, 中国每年产生畜禽粪污约 38 亿 $\mathrm{t}$ 。伴随着种养业发展方式的转变, 种养分离现 象凸显, 粪便还田利用受阻对生态环境造成极大压力 ${ }^{[2]}$ 。国家为防治养殖污染先后颁布 《畜禽养殖业污染物排放标准》《畜禽养殖业污染防治技术政策》《全国畜禽养殖污染防治 “十二五” 规划》《畜禽规模养殖污染防治条例》等政策法规，但畜禽粪污综合利用率仍 然不高，不但没有有效发挥其资源潜力 ${ }^{[3]}$ ，反而成为主要的农业面源污染源之一。众所周 知, 粪污治理问题最终落脚在养殖户的治理行为是否发生, 学者们虽对养殖户的粪污治 理意愿与行为开展了大量研究, 但已有研究忽略了意愿向行为转化的问题, 意愿作为行 为的预测指标, 研究养殖户的粪污治理意愿能够为提高养殖户治理粪污参与率提供理论 指导。然而, 现实中存在两种意愿与行为不一致的情景：一是养殖户有粪污治理意愿却 没有发生治理行为, 若通过该意愿来预测行为, 可能会夸大意愿对行为的影响; 二是养 殖户没有粪污治理意愿却发生了治理行为，这种行为更多的受外界压力的影响而发生， 无法通过养殖户的意愿进行准确预测，并且这些养殖户很有可能退出粪污治理。只有当 养殖户粪污治理意愿与行为一致时, 意愿才更有可能转化为行为, 由此产生行为的养殖 户很有可能从中获得一定的收益, 并且这种行为更具有可持续性。因此, 考察养殖户粪 污治理意愿与行为之间的一致性，对于提高粪污综合利用率具有非常重要的现实意义。

收稿日期：2019-04-20；修订日期：2019-06-29

基金项目：国家社会科学基金重大项目（18ZDA048）；中国工程院重大咨询项目（2018-XZ-15-04）

作者简介：赵俊伟（1986-)，男，河南许昌人，博士研究生，研究方向为农业经济。E-mail: zjw201211@126.com

通讯作者：尹昌斌（1968-)，男，安徽桐城人，博士，研究员，研究方向为农业资源利用、农业经济。

E-mail: yinchangbin@caas.cn 
基于此，本文结合意愿与行为的内在关系，利用吉林、辽宁两省生猪规模养殖户 ${ }^{\circledR}$ 的调研 数据, 对调研样本进行分组 (无意愿无行为、有意愿无行为、有意愿有行为), 从意愿转 化行为的视角对养殖户治理行为的影响因素进行探讨。

\section{1 研究方法与数据来源}

\section{1 理论分析}

环境行为学注重人的行为与环境之间的作用, 并通过各种手段将了解的知识用于环 境质量改善。其中, 计划行为理论（Theory of Planned Behavior, TPB）是环境行为研究 中影响较为广泛的理论模型之一, 该理论由 $A j z e \mathrm{~m}^{[4]}$ 提出, 并且在环境行为领域得到较为 丰富的实证研究和检验。该理论通过期望价值为切人点对个体决策行为进行诠释, 个体 实际行为的发生由其行为意愿决定 ${ }^{[]}$, 行为意愿是个体实际行为最直接的影响因素, 受个 体行为态度、主观规范和感知行为控制的重要影响, 而行为态度、主观规范和感知行为 控制又受到行为信念、规范信念、控制信念等影响 ${ }^{[0]}$ 。行为信念是指个体拥有大量有关行 为可能结果的信念，规范信念是指个 体预期到重要他人或团体对其是否应 该执行某特定行为的期望，控制信念 是指个体知觉到的可能促进和阻碍执 行行为的因素。基于理论分析及文献 研究, 本文在计划行为理论基础上构 建生猪规模养殖粪污治理行为机理形 成框架（图 1)。

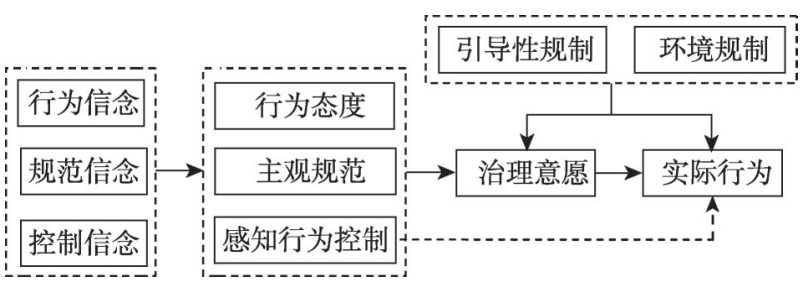

图 1 扩展的计划行为理论模型

Fig. 1 Extended theory of planned behavior

影响养殖户粪污治理意愿的因素很多, 其中, 计划行为理论模型中, 行为态度表征 养殖户对执行污染治理行为的接纳程度。当其对污染治理行为评价是正向的, 则将产生 积极的行为态度。当养殖户感知污染治理行为能够带来居住环境改善、养殖效益提升、 疫病发生率降低等好处时, 将产生积极的行为态度, 有利于增加其外部动机, 将对其污 染治理意愿和污染治理行为产生一定的影响。主观规范表征养殖户对参与污染治理时感 知的社会压力。周围居民对会督促养殖户的污染治理行为。感知行为控制表征养殖户感 知其参与污染治理能够控制并执行治理行为的难易程度。感知行为控制能力越强, 其执 行行为实现的可能性越大 ${ }^{[7]}$, 主要由感知因素和评估因素组成。其中, 感知因素为养殖户 参与污染治理的能力、资源和机会, 在污染治理中对粪污的了解、学习到的相关专业知 识、经济条件等; 评估因素主要体现在养殖户的禀赋效应方面, 养殖户是否愿意并进行 污染治理, 主要取决于治理过程中获得的收益, 只有当养殖户感知自身失去的价值等于 或小于污染治理后获得的价值时, 才能激发污染治理的欲望, 并为此付出行动。此外, 社会经济特征变量、引导性规制和环境规制等相关变量也影响治理意愿与行为的发生。 社会经济特征变量通过行为态度、主观规范和感知行为控制三个因素影响污染治理意愿 和行为 ${ }^{[8]}$, 社会经济特征变量主要包括个人特征, 如年龄 ${ }^{[9]}$ 、文化程度 ${ }^{[10]}$ 、养殖年限 ${ }^{[11]}$

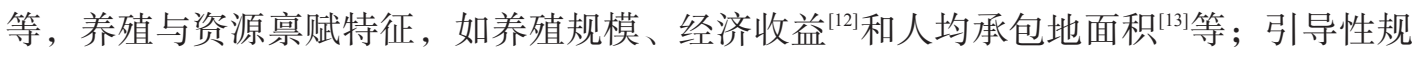

(1) 本文依据《全国农产品成本收益资料汇编》年均存栏 31 100头为小规模、101 1000头为中规模、1000头以 上为大规模, 并统称为生猪规模养殖户。 
制，如对粪污治理的宣传与培训 ${ }^{[14]}$; 环境规制，如以政府补占 ${ }^{[15]}$ 为主的经济激励型和以 政府监管为主的命令控制型环境规制变量等。

行为经济学通过对微观个体行为进行分析发现, 个体行为的意愿是其从事特定行为 的主观概率, 主要表现为参与的意愿程度 ${ }^{[1]}$ 。养殖户的粪污治理意愿是其愿意采取粪污 治理行为的概率大小, 养殖户的粪污治理行为是其对粪污进行处理并实现污染治理目标 所发生的行为。意愿是个体某种行为的心理表现, 是行为发生的前奏。Zeithaml等 ${ }^{[17}$ 认为 意愿和行为之间存在显著相关关系, 意愿主要通过两方面对实际行为产生影响, 包括源 自意愿的承诺和意愿的实现过程, 当意愿达到实际行为的阈值时, 实际行为才能实现。 然而, Wegner ${ }^{[18]}$ 认为意愿与行为的因果关系并不显著。Sheera ${ }^{[19]}$ 通过 Meta分析得出意愿 与行为之间的相关系数为 0.53 , 并进一步论证了意愿与行为直接的差距, 认为意愿的性 能与其相应的基础、目标、时间稳定性及个体执行力等因素影响意愿向行为转化, 同时 行为受到个体的经验、习惯以及外部因素等的影响可能形成自发响应。因此, 意愿与行 为不能总是保持一致。可能存在以下原因与现象：一是由于意愿与行为之间的时间差使 实施行为未来得及实现 ${ }^{[20]}$; 二是意愿受外部条件影响不能达成实际行为, 即 “有意愿无 行为” 的行为转化受阻; 三是主体行为能力受限。结合以上分析可知, 养殖户的污染治 理行为转化过程中存在三种状态，即 “无意愿无行为” “有意愿无行为” 和 “有意愿有行 为”, 并且其行为的发生存在以下路径：“无意愿无行为” $\rightarrow$ “有意愿无行为” $\rightarrow$ “有意 愿有行为” 或者是 “无意愿无行为” $\rightarrow$ “有意愿有行为”。

\section{2 模型构建}

无序多分类Logistic 回归模型，又称多元选择模型。模型中因变量为多项无序分类变 量, 又称名义变量, 其水平数多于 2 个, 因变量水平之间不存在等级递增或递减关系, 对多项无序分类的因变量采用Logistic 回归，是通过拟合广义Logistic 模型的方法进行。

假若因变量有 $n$ 个状态, 首先定义因变量的某一个状态为参照状态, 其他各个状态 与参照状态相比, 拟合 $n-1$ 个 ( $n$ 为因变量个数) 广义 Logistic 回归模型, 本章节中无序 多分类 Logistic 回归模型因变量共有 3 个状态, 分别为 “无意愿无行为” “有意愿无行 为” “有意愿有行为”，分别用 “ 0 ” “ 1 ” “2” 表示，其中， $0 、 1 、 2$ 不表示等级水平，而 是无序变量的数值代码，对应状态取值的概率分别为 $\pi_{0} 、 \pi_{1} 、 \pi_{2}$, 其中, 状态 “ 0 ” 是 其他因变量的共同参照水平。对 $i$ 个自变量拟合模型如下:

$$
\begin{aligned}
& \log \text { it } \pi_{0}=\ln \left(\frac{\pi_{0}}{\pi_{0}}\right)=0 \\
& \log \text { it } \pi_{1}=\ln \left(\frac{\pi_{1}}{\pi_{0}}\right)=\alpha_{1}+\beta_{11} X_{1}+\beta_{12} X_{2}+\cdots+\beta_{1 i} X_{i} \\
& \log \text { it } \pi_{2}=\ln \left(\frac{\pi_{2}}{\pi_{0}}\right)=\alpha_{1}+\beta_{21} X_{1}+\beta_{22} X_{2}+\cdots+\beta_{2 i} X_{i} \\
& \pi_{j}=P(Y=j \mid X)=\left\{\begin{array}{l}
\frac{1}{1+\sum_{k=2}^{J} \exp \left(\beta_{k} X\right)}(j=0) \\
\frac{\exp \left(\beta_{j} X\right)}{1+\sum_{k=2}^{J} \exp \left(\beta_{k} X\right)}(j=1,2)
\end{array}\right.
\end{aligned}
$$

\section{3 数据来源}

数据来源于 2017 年 9 月至 2018 年 1 月在吉林、辽宁两省的生猪调出大县开展的实地 
问卷调查，结合 “南猪北移” 的发展趋势，两省作为生猪养殖潜力增长区，粪污处理问 题也成为生猪生产可持续发展的关键，综合考虑调研实施可行性的限制及数据资料的可 获得性, 选择吉林、辽宁两省作为本文的研究区域。调查地域范围涉及吉林省 4 市 11 县、 辽宁省 5 市 14 县。调查对象为生猪规模养殖户。由于养殖户分布较为分散, 并且受疫病 防控以及养殖户的受访便利条件等因素的制约, 通过随机抽样获得的样本养殖户可能出现 拒绝受访或者不能到指定地点受访, 调查样本很难做到完全随机抽样, 因此由当地畜牧业 相关负责人协助进行。调查采用实地调研与集中调研相结合方式，调研员与养殖户主一对 一进行问卷访谈, 调研成员由博士生和硕士生组成。本次调研共获得 1200 份养殖户问卷, 剔除缺失和极端数据的无效问卷之后，得到有效问卷 1124 份，问卷有效率达 $93.7 \%$ 。

\section{4 样本基本特征}

如表 1 所示，从总样本看：（1）被调查养殖户主涵盖不同的年龄结构，31 60 岁年龄 段居多, 其中较为集中于 $41 \sim 50$ 岁, 占总样本的 $45.73 \%$, 分布在 31 40 岁和 51 60岁的养 殖户数量大致相当, 分别占总样本的 $21.35 \%$ 和 $23.67 \% ; 30$ 岁及以下的和 60 岁以上的养 殖户分别占总样本的 $4 \%$ 和 $5.25 \%$; 从不同规模角度看, 养殖户主的年龄结构也有所差 异，其中，小规模养殖户和中等规模养殖户多集中在 41 50 岁，分别占 49.8\% 和 $45.85 \%, 30$ 岁及以下和 60 岁以上这两个年龄段占比较低。(2) 从文化程度来看, 养殖户 主的文化程度普遍偏低, 具有高中及以上学历的仅占 $21.44 \%$; 从不同规模角度看, 小规 模养殖户和中规模养殖户文化程度多为高中以下，大规模养殖户文化程度相对较高，具 有高中及以上文化程度的养殖户占 $50 \%$ 。(3) 从养殖年限看, 具有 11 20年养殖年限的 规模养殖户占 $46.89 \%, 10$ 年以下的占 $35.05 \%$ ，均高于具有 20 年以上的所占的比例 (18.06\%)。从不同规模角度看，小规模和中规模养殖户的养殖年限集中在 11 20年，分 别占 $45.82 \%$ 和 $48.22 \%$; 大规模养殖户的养殖年限以 10 年及以下和 $11 \sim 20$ 年为主, 分别占 $43.86 \%$ 和 $40.35 \%$, 在 20 年以上的偏少，仅占 $15.79 \%$ 。（4）从养殖规模看，中规模养猪 户占比最高, 占总样本量的 $67.53 \%$, 均高于小规模和大规模养猪户所占比例, 在一定程

表 1 样本的基本信息

Table 1 The basic information of samples

\begin{tabular}{|c|c|c|c|c|c|c|c|c|c|}
\hline \multirow{2}{*}{ 类型 } & \multirow{2}{*}{ 选项 } & \multicolumn{2}{|c|}{ 总样本 } & \multicolumn{2}{|c|}{ 小规模 } & \multicolumn{2}{|c|}{ 中规模 } & \multicolumn{2}{|c|}{ 大规模 } \\
\hline & & 样本数/个 & 比例 $/ \%$ & 样本数/个 & 比例 $/ \%$ & 样本数/个 & 比例 $/ \%$ & 样本数/个 & 比例 $/ \%$ \\
\hline \multirow[t]{2}{*}{ 地区 } & 吉林省 & 522 & 46.44 & 69 & 27.49 & 395 & 52.04 & 58 & 50.88 \\
\hline & 辽宁省 & 602 & 53.56 & 182 & 72.51 & 364 & 47.96 & 56 & 49.12 \\
\hline \multirow[t]{5}{*}{ 年龄/岁 } & 30 及以下 & 45 & 4.00 & 9 & 3.59 & 27 & 3.56 & 9 & 7.89 \\
\hline & $31 \sim 40$ & 240 & 21.35 & 40 & 15.94 & 175 & 23.06 & 25 & 21.93 \\
\hline & $41 \sim 50$ & 514 & 45.73 & 125 & 49.8 & 348 & 45.85 & 41 & 35.96 \\
\hline & $51 \sim 60$ & 266 & 23.67 & 63 & 25.1 & 171 & 22.53 & 29 & 25.44 \\
\hline & 60 以上 & 59 & 5.25 & 14 & 5.58 & 38 & 5.01 & 10 & 8.77 \\
\hline \multirow[t]{2}{*}{ 文化程度 } & 高中以下 & 883 & 78.56 & 221 & 88.05 & 605 & 79.71 & 57 & 50 \\
\hline & 高中及以上 & 241 & 21.44 & 30 & 11.95 & 154 & 20.29 & 57 & 50 \\
\hline \multirow[t]{3}{*}{ 养殖年限/年 } & 10 及以下 & 394 & 35.05 & 91 & 36.25 & 253 & 33.33 & 50 & 43.86 \\
\hline & $11 \sim 20$ & 527 & 46.89 & 115 & 45.82 & 366 & 48.22 & 46 & 40.35 \\
\hline & 20 以上 & 203 & 18.06 & 45 & 17.93 & 140 & 18.45 & 18 & 15.79 \\
\hline
\end{tabular}


度上表明吉林和辽宁两省的中规模养猪户已经成为生猪养殖业的中坚力量。

\section{5 变量选取与描述性统计}

根据前文分析及相关理论，从 7 个维度构建生猪规模养殖粪污治理意愿与行为一致 性的自变量（表 2)。由于因变量涉及 “无意愿无行为” “有意愿无行为” “有意愿有行 为” 三种状态，因此，本文用于分析的样本量为 903 ，其中有意愿的样本量为 587 , “有 意愿无行为”, 即意愿与行为不一致的样本量为 242 , 占 $26.80 \%$; “有意愿有行为”, 即意 愿与行为一致的样本量为 345 , 占 $38.21 \%$ 。具体变量说明与样本描述性统计如下:

\section{表 2 变量含义与描述性统计分析}

Table 2 Variable and descriptive statistical analysis

\begin{tabular}{|c|c|c|c|c|}
\hline \multicolumn{2}{|c|}{ 变量 } & \multirow{2}{*}{$\begin{array}{c}\text { 定义及赋值 } \\
\text { 受访者实际年龄 }\end{array}$} & \multirow{2}{*}{$\begin{array}{c}\text { 均值 } \\
46.394\end{array}$} & \multirow{2}{*}{$\begin{array}{c}\text { 标准差 } \\
8.569\end{array}$} \\
\hline 个体特征 & 年龄 & & & \\
\hline & 文化程度 & 高中以下 $=0$; 高中及以上 $=1$ & 0.224 & 0.417 \\
\hline & 养殖年限 & 从事养猪业时间/年 & 13.663 & 6.615 \\
\hline \multirow[t]{6}{*}{ 养殖与资源禀赋特征 } & 养殖规模 & 小规模: 否 $=0 ;$ 是 $=1$ & 0.254 & 0.435 \\
\hline & & 中规模: 否 $=0 ;$ 是 $=1$ & 0.649 & 0.478 \\
\hline & & 大规模: 否 $=0$; 是 $=1$ & 0.097 & 0.297 \\
\hline & 与村庄距离 & 猪场与村庄的距离 $/ \mathrm{m}$ & 274.867 & 486.315 \\
\hline & 粪污消纳地面积 & 养殖户经营农田面积/亩 & 32.773 & 56.038 \\
\hline & 养殖净收益 & 每头猪的年均净收益/（元/头） & 217.125 & 111.780 \\
\hline \multirow[t]{3}{*}{ 行为态度 } & 周围环境污染认知 & 不污染 $=0 ;$ 污染 $=1$ & 0.734 & 0.442 \\
\hline & 对猪生长影响认知 & 没有影响 $=0$; 有影响 $=1$ & 0.731 & 0.444 \\
\hline & 对人体健康影响认知 & 没有影响 $=0$; 有影响 $=1$ & 0.736 & 0.441 \\
\hline 主观规范 & 周边群众舆论 & 没有意见 $=0$; 有意见 $=1$ & 0.329 & 0.470 \\
\hline \multirow[t]{2}{*}{ 感知行为控制 } & 粪污处理技术 & 不了解 $=0 ;$ 了解 $=1$ & 0.422 & 0.494 \\
\hline & 粪污处理经济条件 & 不具备 $=0$; 具备 $=1$ & 0.400 & 0.490 \\
\hline \multirow[t]{2}{*}{ 引导性规制 } & 粪污治理宣传 & 没有 $=0 ;$ 有 $=1$ & 0.566 & 0.496 \\
\hline & 粪污处理相关培训 & 没有 $=0 ;$ 有 $=1$ & 0.352 & 0.478 \\
\hline \multirow[t]{2}{*}{ 环境规制 } & 政府补贴 & 没有 $=0 ;$ 有 $=1$ & 0.136 & 0.343 \\
\hline & 政府监管 & 没有 $=0$; 有 $=1$ & 0.736 & 0.441 \\
\hline
\end{tabular}

一是个体特征，包括年龄、文化程度、养殖年限。二是养殖与资源禀赋特征，包括 养殖规模、猪场与村庄的距离、粪污消纳地面积、养殖净收益。其中，粪污消纳地面积 以养殖户经营的农田面积来衡量，包括通过土地流转、租赁等形式获得使用权的农田。 三是行为态度，主要包括周围环境污染认知、对猪生长影响认知以及对人体健康影响认 知。分别以 “粪便处理不当是否造成周边环境污染” “粪便污染对猪的健康生长是否有不 利影响” “粪便污染对人的身体健康是否有影响” 来测量。四是主观规范，主要包括周边 群众與论。调查问题是 “周边群众是否因粪便污染环境问题向您提出过意见”。五是感知 行为控制，主要包括粪污处理技术、粪污处理经济条件。其中粪污处理技术以“您是否 了解粪污处理技术? 例如：粪污的无害化处理及其肥料化或能源化等处理技术”; 粪污处 理经济条件以 “您是否具备粪污处理的经济条件? 包括粪污处理设施建设及相关设备的 购买与运行”。六是引导性规制，主要包括粪污治理宣传、粪污处理相关培训。分别以 “政府相关部门是否对于粪污治理进行过宣传? 如通过新闻媒体、网络信息平台或宣传单 
页等形式开展宣传活动” “政府相关部门是否针对粪污处理组织开展学习培训” 来测量。 七是环境规制, 主要包括以政府补贴为代表的经济激励型环境规制和以政府监管为代表 的命令控制型环境规制，调查问题分别是 “您家猪场在粪污处理方面是否享受过政府补 贴” 和“政府部门是否对您家猪场的粪污处理问题进行过监督检查”。

\section{6 生猪规模养殖粪污治理意愿与行为一致性分析}

从养殖户的粪污治理意愿与行为一致性特征来看（表 3), 在年龄分布上，41 50 岁 的养殖户中, “有意愿无行为” 和 “有意愿有行为” 的样本占比最高, 年龄在 30 岁以下 的养殖户中的这一比例最低; 从文化程度看, 具有高中以下学历的养殖户占比较高, 并 且 “有意愿无行为” 的养殖户占比多于 “有意愿有行为” 的养殖户; 从养殖年限看, 养 殖年限在 11 20年的养猪户中, 在 “有意愿无行为” 和 “有意愿有行为” 的样本中均占比 最高，并且在养殖年限 20 年以上的样本中，这一比例均较低；从养殖规模看，中规模养殖 户中, 在 “有意愿无行为” 和 “有意愿有行为” 的样本中均占比最高, 并且在中规模和大 规模养殖户中, 一致性样本量占比高于不一致性的样本量, 尤其在大规模养殖户中较为显 著，而在小规模养殖户中，一致性样本量明显低于不一致的样本量。

表 3 粪污治理意愿与行为一致性统计描述结果

Table 3 Statistical descriptive results of consistency between manure disposal willingness and behavior

\begin{tabular}{|c|c|c|c|c|c|c|c|}
\hline \multirow{2}{*}{ 变量 } & \multirow{2}{*}{ 选项 } & \multicolumn{2}{|c|}{ 有意愿 } & \multicolumn{2}{|c|}{ 有意愿无行为 (不一致) } & \multicolumn{2}{|c|}{ 有意愿有行为 (一致) } \\
\hline & & 个数 $/$ 个 & 比例/\% & 个数/个 & 比例/\% & 个数 $/$ 个 & 比例 $/ \%$ \\
\hline \multirow[t]{5}{*}{ 年龄/岁 } & 30 及以下 & 18 & 3.07 & 2 & 0.83 & 16 & 4.64 \\
\hline & $31 \sim 40$ & 118 & 20.10 & 50 & 20.66 & 68 & 19.71 \\
\hline & $41 \sim 50$ & 284 & 48.38 & 119 & 49.17 & 165 & 47.83 \\
\hline & $51 \sim 60$ & 134 & 22.83 & 60 & 24.79 & 74 & 21.45 \\
\hline & 60 以上 & 33 & 5.62 & 11 & 4.55 & 22 & 6.38 \\
\hline \multirow[t]{2}{*}{ 文化程度 } & 高中以下 & 434 & 73.94 & 195 & 80.58 & 239 & 69.28 \\
\hline & 高中及以上 & 153 & 26.06 & 47 & 19.42 & 106 & 30.72 \\
\hline \multirow[t]{3}{*}{ 养殖年限/年 } & 10 及以下 & 205 & 34.92 & 79 & 32.64 & 126 & 36.52 \\
\hline & $11 \sim 20$ & 279 & 47.53 & 112 & 46.28 & 167 & 48.41 \\
\hline & 20 以上 & 103 & 17.55 & 51 & 21.07 & 52 & 15.07 \\
\hline \multirow[t]{3}{*}{ 养殖规模 } & 小规模 & 117 & 19.93 & 69 & 28.51 & 48 & 13.91 \\
\hline & 中规模 & 403 & 68.65 & 164 & 67.77 & 239 & 69.28 \\
\hline & 大规模 & 67 & 11.41 & 9 & 3.72 & 58 & 16.81 \\
\hline \multicolumn{2}{|c|}{ 样本量 } & \multicolumn{2}{|c|}{587} & \multicolumn{2}{|c|}{242} & \multicolumn{2}{|c|}{345} \\
\hline
\end{tabular}

\section{2 结果分析}

\section{1 意愿与行为一致性的影响因素: 相关性分析}

为探讨显著影响养殖户治理意愿与行为一致性的因素, 通过计算因变量和自变量之 间的皮尔逊相关系数进行分析。从表 4 中可以看出, 文化程度、大规模养殖、猪场与村 庄距离、粪污消纳地面积、养殖净收益、粪污处理技术、粪污处理经济条件、粪污治理 宣传、粪污处理相关培训、政府补贴、政府监管等 11 个变量均在 $1 \%$ 显著性水平上和养 殖户的粪污治理意愿与行为一致性显著正相关，而养殖规模特征变量中的小规模养殖和 
表 4 相关性分析结果

Table 4 The relevant analysis results

\begin{tabular}{lc||lc}
\hline \multicolumn{1}{c||}{ 自变量 } & 皮尔逊相关系数 & \multicolumn{1}{c}{ 自变量 } & \multicolumn{1}{c}{ 皮尔逊相关系数 } \\
\hline 年龄 & -0.023 & 对猪生长影响认知 & 0.031 \\
文化程度 & $0.127^{* * * *}$ & 对人体健康影响认知 & 0.043 \\
养殖年限 & -0.035 & 周边群众舆论 & -0.063 \\
小规模 & $-0.180^{* * * *}$ & 粪污处理技术 & $0.327^{* * *}$ \\
中规模 & 0.016 & 粪污处理经济条件 & $0.196^{* * *}$ \\
大规模 & $0.203^{* * * *}$ & 粪污治理宣传 & $0.308^{* * * *}$ \\
猪场与村庄距离 & $0.185^{* * * *}$ & 粪污处理相关培训 & $0.349^{* * * *}$ \\
粪污消纳地面积 & $0.152^{* * * *}$ & 政府补贴 & $0.382^{* * * *}$ \\
养殖净收益 & $0.182^{* * * *}$ & 政府监管 & $0.304^{* * * *}$ \\
周围环境污染认知 & 0.040 & & \\
\hline
\end{tabular}

注: “”表示 $1 \%$ 的统计显著性水平。

养殖户的粪污治理意愿与行为一致性显著负相关。而养殖户的年龄、养殖年限、周围环 境污染认知、对猪生猪影响认知、对人体健康影响认知、周边群众與论这 6 个变量和养 殖户的粪污治理意愿与行为一致性的相关程度较小。

\section{2 意愿与行为一致性的影响因素:Logistic 回归分析}

以上对治理意愿与治理行为一致性影响因素的相关性分析只是检验了自变量与因变 量之间的相关关系的显著性及作用方向，而影响养殖户进行粪污治理意愿与行为一致性 的因素之间可能存在相互作用，基于此，运用计量模型进一步对影响意愿与行为一致性 的因素进行分析。结合前文对相关因变量和自变量的分类与定义，模型 I 和模型 II 以 “无 意愿无行为” 作为参照组, 分别分析 “有无意愿” 和 “有无行为” 的影响因素; 模型 III 以 “有意愿无行为” 作为参照组, 分析意愿转化行为的影响因素。并运用多分类Logistic 回归分析方法对模型进行拟合。

\subsection{1 模型检验}

从表 5 可以看出, 当 “无意愿无行为”, 即 “ 0 ” 作为参照时, 模型引人常数项后, 对数似然值从 1964.814 减少至 $1435.540, P$ 值为 0 , 表明至少有一个解释变量的偏回归系 数不等于 0 , 模型具有统计意义, 用于检验假设的统计量具有可靠性; 同理, 当 “有意 愿无行为”, 即 “1” 作为参照时，同样满足以上条件，具有统计意义。

拟合优度检验结果显示 (表 6), 皮尔逊与偏差相关系数对应的 $P$ 值均大于 0.05 , 进 一步验证了模型拟合良好。

表 5 模型拟合信息

Table 5 The fitting information of model

\begin{tabular}{clcccc}
\hline 参照组 & \multicolumn{1}{c}{ 模型形式 } & 对数似然值 & 卡方值 & 自由度 & 显著性 \\
\hline “无意愿无行为” & 只有常数项的模型 & 1964.814 & - & - & - \\
& 最终模型 & 1435.540 & 529.274 & 36 & 0.000 \\
“有意愿无行为” & 只有常数项的模型 & 795.588 & - & - & - \\
& 最终模型 & 585.284 & 210.304 & 18 & 0.000 \\
\hline
\end{tabular}


表 6 拟合优度检验

Table 6 The goodness of fit test

\begin{tabular}{ccccc}
\hline 参照组 & 相关系数 & 卡方 & 自由度 & 显著性 \\
\hline “无意愿无行为” & 皮尔逊 & 1736.312 & 1768 & 0.700 \\
& 偏差 & 1435.540 & 1768 & 1.000 \\
“有意愿无行为” & 皮尔逊 & 544.130 & 568 & 0.758 \\
& 偏差 & 585.284 & 568 & 0.299 \\
\hline
\end{tabular}

\section{2 .2 结果分析}

回归结果分为三类，第一类为养殖户 “有无意愿” 的回归结果（模型 I），主要分析 影响养殖户治理意愿形成的因素；第二类为养殖户 “有无行为” 的回归结果（模型 II）, 主要分析影响养殖户治理行为形成的因素; 第三类为养殖户 “意愿转化行为” 的回归结 果 (模型 III), 主要分析养殖户在具有治理意愿的前提下，促使其意愿向行为转化的影 响因素，具体如表 7 所示。

（1）个体特征。养殖户的年龄对于其粪污治理意愿与行为具有正向影响，但对于其 意愿向行为转化影响不显著, 甚至阻碍其意愿向行为的转化; 养殖年限对养殖户粪污治 理行为及意愿转化行为具有显著的负向影响, 表明养殖年限越长, 养殖户的治理意愿与

表 7 模型回归结果

Table 7 The regression results of the model

\begin{tabular}{|c|c|c|c|c|c|c|c|c|c|}
\hline \multirow[t]{2}{*}{ 类别 } & \multicolumn{3}{|c|}{$\begin{array}{c}\text { 有无意愿 (模型I） } \\
\text { (有意愿无行为/无意愿无行为) }\end{array}$} & \multicolumn{3}{|c|}{$\begin{array}{c}\text { 有无行为 (模型 II ) } \\
\text { (有意愿有行为/无意愿无行为) }\end{array}$} & \multicolumn{3}{|c|}{$\begin{array}{c}\text { 意愿转化行为 (模型 III) } \\
\text { (有意愿有行为/有意愿无行为) }\end{array}$} \\
\hline & $B$ & 标准误 & $\operatorname{Exp}(B)$ & $B$ & 标准误 & $\operatorname{Exp}(B)$ & $B$ & 标准误 & $\operatorname{Exp}(B)$ \\
\hline 截距 & 1.422 & 1.141 & & $5.424^{* * *}$ & 1.121 & & $3.860^{* * *}$ & 0.992 & \\
\hline 年龄 & $0.023^{*}$ & 0.012 & 1.023 & $0.022^{*}$ & 0.013 & 1.023 & -0.003 & 0.013 & 0.997 \\
\hline 文化程度 $=0$ & -0.256 & 0.249 & 0.774 & -0.430 & 0.273 & 0.650 & -0.124 & 0.261 & 0.883 \\
\hline 养殖年限 & -0.018 & 0.015 & 0.983 & $-0.046^{* * *}$ & 0.017 & 0.955 & $-0.032^{* *}$ & 0.016 & 0.968 \\
\hline 小规模 $=0$ & 0.081 & 0.222 & 1.085 & $0.508^{*}$ & 0.272 & 1.663 & $0.522^{*}$ & 0.273 & 1.686 \\
\hline 大规模 $=0$ & 0.006 & 0.512 & 1.006 & -0.164 & 0.511 & 0.849 & 0.001 & 0.460 & 1.001 \\
\hline 与村庄距离 & $-0.001^{* * *}$ & 0.000 & 0.999 & $-0.001^{* * *}$ & 0.000 & 0.999 & -0.0002 & 0.000 & 1.000 \\
\hline 粪污消纳地面积 & $0.007^{* *}$ & 0.003 & 1.007 & $0.013^{* * *}$ & 0.004 & 1.013 & $0.005^{* *}$ & 0.003 & 1.005 \\
\hline 养殖净收益 & 0.001 & 0.001 & 1.001 & $0.003^{* * *}$ & 0.001 & 1.003 & $0.002^{* *}$ & 0.001 & 1.002 \\
\hline 周围环境污染认知 $=0$ & $-0.625^{* * *}$ & 0.229 & 0.535 & $-0.704^{* * *}$ & 0.259 & 0.495 & -0.104 & 0.271 & 0.901 \\
\hline 对猪生长影响认知 $=0$ & $-0.699^{* * *}$ & 0.261 & 0.497 & $-0.502^{*}$ & 0.300 & 0.605 & 0.150 & 0.321 & 1.162 \\
\hline 对人体健康影响认知 $=0$ & -0.376 & 0.267 & 0.687 & $-0.734^{* *}$ & 0.312 & 0.480 & -0.340 & 0.329 & 0.712 \\
\hline 周边群众舆论 $=0$ & $-0.649^{* * * *}$ & 0.216 & 0.522 & $-0.974^{* * *}$ & 0.246 & 0.378 & $-0.414^{*}$ & 0.234 & 0.661 \\
\hline 粪污处理技术 $=0$ & -0.299 & 0.220 & 0.741 & $-0.961^{* * *}$ & 0.230 & 0.382 & $-0.650^{* * *}$ & 0.220 & 0.522 \\
\hline 粪污处理经济条件 $=0$ & -0.312 & 0.213 & 0.732 & $-0.706^{* * *}$ & 0.227 & 0.494 & $-0.389^{*}$ & 0.215 & 0.678 \\
\hline 粪污治理宣传 $=0$ & -0.358 & 0.222 & 0.699 & $-0.646^{* * * *}$ & 0.246 & 0.524 & -0.381 & 0.240 & 0.683 \\
\hline 粪污处理相关培训 $=0$ & $-0.430^{*}$ & 0.255 & 0.651 & $-1.047^{* * *}$ & 0.255 & 0.351 & $-0.653^{* * *}$ & 0.240 & 0.521 \\
\hline 政府补贴 $=0$ & -0.559 & 0.780 & 0.572 & $-3.212^{* * *}$ & 0.665 & 0.040 & $-2.649^{* * *}$ & 0.495 & 0.071 \\
\hline 政府监管 $=0$ & -0.376 & 0.237 & 0.686 & $-1.294^{* * *}$ & 0.308 & 0.274 & $-0.954^{* * *}$ & 0.303 & 0.385 \\
\hline
\end{tabular}

注: “.”、“、”分别表示 $1 \% 、 5 \% 、 10 \%$ 的统计显著性水平。 
治理行为一致性的概率越小, 越不利于粪污治理行为的发生。

(2) 养殖与资源禀赋特征。小规模对于治理行为和意愿转化行为具有显著正向影 响, 其中, 小规模的意愿转化行为偏回归系数为 0.522 , 并在 $10 \%$ 显著性水平下通过检 验, 表明对于小规模养殖户，其参与粪污治理意愿与行为一致性的概率相对于其他规模 较高。粪污消纳地面积对养殖户的治理意愿、行为影响显著, 且对于意愿转化行为具有 显著促进作用, 养殖净收益对养殖户的治理行为显著影响, 同样对意愿转化行为具有显 著促进作用, 其中, 粪污消纳地面积和养殖净收益的意愿转化行为系数分别是 0.005 和 0.002 , 均在 $5 \%$ 显著性水平下通过检验，表明养殖户经营的农民面积越大、养殖净收益 越高, 养殖户进行粪污治理意愿与行为一致性的概率就越大，并且越有利于粪污治理意 愿的实现。

（3）行为态度。周围环境污染认知、对猪生猪影响认知的意愿与行为的偏回归系数 为负值, 并且均通过显著性检验, 表明养殖户对粪便污染环境的认知以及对猪生长影响 的认知有利于激发其治理意愿和行为的发生, 并且对意愿转化行为具有一定的促进作 用，但影响不显著。

（4）主观规范。周边群众舆论对养殖户的粪污治理意愿、行为均有显著影响，对意 愿转化行为的偏回归系数为 -0.414 , 表明群众舆论监督越强烈, 养殖户的治理意愿与行 为一致性的概率越高, 越有利于粪污治理行为的发生。

(5) 感知行为控制。粪污处理技术对意愿转化行为的偏回归系数为 -0.65 , 表明养殖 户对粪污处理技术越了解，其治理意愿与行为一致性的概率越高；粪污处理经济条件的 偏回归系数为 -0.389 , 表明若养殖户具备粪污处理经济条件, 其治理意愿与行为一致性 的概率将有所提高。

（6）引导性规制。粪污处理相关培训对意愿转化行为的偏回归系数为 -0.653 , 并且 在 $1 \%$ 显著性水平下通过检验, 表明加强对养殖户的粪污处理相关培训将能提高粪污治理 意愿与行为的概率, 并促进意愿向行为的转化。

（7）环境规制。政府补贴和政府监管对意愿转化行为的偏回归系数分别为 -2.649 和- 0.954 , 均在 $1 \%$ 显著性水平下通过检验，表明政府补贴、政府监管能够提高养殖户治 理意愿与行为一致性的概率。Exp值表明，在其他条件不变的条件下，每增加一单位政 府补贴或政府监管力度, 养殖户的治理意愿与行为一致性的概率将分别增加至原来的 0.071 倍和 0.385 倍, 在一定程度上表明命令控制型环境规制作用强于经济激励型环境规 制的作用。

\section{3 结论与讨论}

利用吉林、辽宁 2 省 25 县的生猪规模养殖户调查数据，基于意愿转化行为视角，通 过对样本进行分组（无意愿无行为、有意愿无行为、有意愿有行为）, 运用无序多分类 Logistic 回归模型从养殖户的个体特征、养殖与资源禀赋特征、行为态度、主观规范、感 知行为控制、引导性规制和环境规制等 7 个维度对养殖户的粪污治理行为影响因素进行 分析。得出如下结论:

（1）养殖户的粪污治理意愿与实际治理行为之间存在一定差异, 意愿与行为不一致 
比率达 $26.8 \%$ 。

（2）养殖户粪污治理意愿受年龄、猪场与村庄距离、粪污消纳地面积、周围环境污染 认知、对猪生猪影响认知、周边群众舆论、粪污处理相关培训等因素的显著影响; 养殖户 粪污治理行为除了受以上意愿影响因素以外，还受养殖年限、养殖规模、养殖净收益、粪 污处理技术、粪污处理经济条件、粪污治理宣传、政府补贴和政府监管等因素的影响。

（3）影响养殖户粪污治理意愿转化行为的因素主要与养殖规模、粪污消纳地面积、 养殖净收益、周边群众與论、粪污处理技术、粪污处理经济条件、粪污处理相关培训、 政府补贴和政府监管等相关性较大，并且对于意愿转化行为具有显著的促进作用，而养 殖年限成为养殖户粪污治理意愿转化行为的阻碍因素。

上述结论揭示了影响生猪规模养殖粪污治理意愿与行为决策的关键因素及其差异。 基于以上结论可得出以下几点启示:

（1）养殖收人是影响养殖户进行粪污治理的重要因素。养殖收人作为养殖户进行粪 污治理的基础条件，只有其达到一定水平，才有意愿和能力进行粪污治理。因此，应通 过各种有效措施保障养殖户的养殖收人水平，进而促进其实施粪污治理。

（2）进一步加大粪污治理宣传培训力度。提高养殖户对粪便污染环境及人畜健康影 响的认知, 强化养殖户保护环境的责任意识; 积极开展粪污处理技术培训, 提升养殖户 粪污治理能力与手段，激发养殖户的治理意愿，进而提升实施治理行为发生的可能性。

（3）有效发挥政府监管与群众舆论作用，加强主观规范。充分利用周边群众社会舆 论等非正式监督功能, 依据粪污治理行为发生机理, 结合内在引导和外在激励手段, 促 进治理意愿向治理行为转化, 将粪污治理内化为养殖户的自主行为。

\section{参考文献(References):}

[1] 赵俊伟, 陈永福, 余乐, 等. 中国生猪养殖业地理集聚时空特征及影响因素. 经济地理, 2019, 39(2): 180-189. [ZHAO J W, CHEN Y F, YU L, et al. Spatial-temporal characteristics and affecting factors of swine breeding industry in China. Economic Geography, 2019, 39(2): 180-189.]

[2] 邱乐丰, 龙文莉, 方豪, 等. 基于种养平衡的杭州市畜禽养殖环境承载力研究. 自然资源学报, 2016, 31(8): 14101419. [QIU L F, LONG W L, FANG H, et al. Regional environmental carrying capacity for livestock and poultry breeding based on planting-breeding balance in Hangzhou city. Journal of Natural Resources, 2016, 31(8): 1410-1419.]

[3] 赵俊伟, 尹昌斌. 青岛市畜禽粪便排放量与肥料化利用潜力分析. 中国农业资源与区划, 2016, 37(7): 108-115. [ZHAO J W, YIN C B. Analysis on the total amount of domestic animal excrement and the potential of fertilizer utilization in Qingdao city. Chinese Journal of Agricultural Resources and Regional Planning, 2016, 37(7): 108-115.]

[4] AJZEM I. The theory of planned behavior. Organizational Behavior and Human Decision Processes, 1991, 50(2): 179211.

[5] 段文婷, 江光荣. 计划行为理论述评. 心理科学进展, 2008, 16(2): 315-320. [DUAN W T, JIANG G R. A review of the theory of planned behavior. Advances in Psychological Science, 2008, 16(2): 315-320.]

[6] KIRIAKIDIS S P. Application of the theory of planned behavior to recidivism: The role of personal norm in predicting behavioral intentions of re-offending 1. Journal of Applied Social Psychology, 2010, 38(9): 2210-2221.

[7] KRAFT P, RISE J, SUTTON S, et al. Perceived difficulty in the theory of planned behaviour: Perceived behavioural control or affective attitude?. Br J Soc Psychol, 2005, 44(3): 479-496.

[8] SIMON F. Consumer adoption of No Junk Mail stickers: An extended planned behavior model assessing the respective role of store flyer attachment and perceived intrusiveness. Journal of Retailing \& Consumer Services, 2016, $29:$ 12-21. 
[9] 蒋否, 张俊䒊, 何可. 基于农户兼业视角的农业废弃物资源循环利用意愿及其影响因素比较: 以湖北省为例. 长江流 域资源与环境, 2014, 23(10): 1432-1439. [JIANG L, ZHANG J B, HE K. Comparisons of farmers' willingness to recycle resources of agricultural waste and Influencing factors in the perspective of farmers' concurrent business: An empirical evidence from Hubei province. Resources and Environment in the Yangtze Basin, 2014, 23(10): 1432-1439.]

[10] 宾幕容, 覃一枝, 周发明. 湘江流域农户生猪养殖污染治理意愿分析. 经济地理, 2016, 36(11): 154-160. [BIN M R, QING Y Z, ZHOU F M. Influencing factors and hierarchy of farmers' willingness on pig breeding pollution control in Xiangjiang River Basin: Based on investigation of 367 pig farmers. Economic Geography, 2016, 36(11): 154-160.]

[11] 陈诗波, 王亚静, 樊丹. 基于农户视角的乡村清洁工程建设实践分析: 来自湖北省的微观实证. 中国农村经济, 2009, (4): 62-71. [CHEN S B, WANG Y J, FAN D. Practice analysis of rural clean engineering construction from the perspective of peasant households: A micro-empirical study from Hubei province. Chinese Rural Economy, 2009, (4): 62-71.]

[12] 朱哲毅, 应瑞瑶, 周力. 畜禽养殖末端污染治理政策对养殖户清洁生产行为的影响研究: 基于环境库兹涅茨曲线视 角的选择性试验. 华中农业大学学报: 社会科学版, 2016, (5): 55-62, 145. [ZHU Z Y, YING R Y, ZHOU L. The research on the effect of policy concerning terminal pollution in livestock breeding on farmers clean production behavior: A choice experiment based on the EKC perspective. Journal of Huazhong Agricultural University: Social Sciences Edition, 2016, (5): 55-62, 145.]

[13] 穆亚丽, 冯淑怡, 马力, 等. 农户沼肥还田决策行为及其经济效应评价. 自然资源学报, 2017, 32(10): 1678-1690. [MU Y L, FENG S Y, MA L, et al. Farm household's decision of returning biogas residues to farmland and its economic effects. Journal of Natural Resources, 2017, 32(10): 1678-1690.]

[14] 孔凡斌, 张维平, 潘丹. 基于规模视角的农户畜昺养殖污染无害化处理意愿影响因素分析: 以 5 省 754 户生猪养殖 户为例. 江西财经大学学报, 2016, (6): 75-81. [KONG F B, ZHANG W P, PAN D. Analysis on the influencing factors of farmers' willingness to deal with the pollution of livestock and poultry breeding from the perspective of scale: A case study of 754 livestock and poultry breeders in 5 provinces. Journal of Jiangxi University of Finance and Economics, 2016, (6): 75-81.]

[15] 张玉梅, 乔娟. 生态农业视角下养猪场(户)环境治理行为分析: 基于北京郊区养猪场(户)的调研数据. 技术经济, 2014, 33(7): 75-81. [ZHANG Y M, QIAO J. Analysis on environmental governance behavior of pig farm from perspective of ecological agriculture: Based on survey data of pig farms in suburb of Beijing. Technology Economics, 2014, 33 (7): 75-81.]

[16] ARMITAGE C J, CONNER M. Efficacy of the theory of planned behaviour: A meta-analytic review. British Journal of Social Psychology, 2001, 40(4): 471-499.

[17] ZEITHAML V A, BERRY L L, PARASURAMAN A. The behavioral consequences of service quality. Journal of Marketing, 1996, 60(2): 31-46.

[18] WEGNER D M. The illusion of conscious will. Mit Pr, 2003, 159(2): 197-213.

[19] SHEERAN P. Intention-behavior relations: A conceptual and empirical review. European Review of Social Psychology, 2002: 12 .

[20] CONNER M. Prospective prediction of health-related behaviours with the theory of planned behaviour: A meta-analysis. Health Psychology Review, 2011, 5(2): 97-144. 


\title{
Analysis on influencing factors of manure pollution treatment in scale pig breeding: Based on the perspective of willingness-to-behavior transformation
}

\author{
ZHAO Jun-wei ${ }^{1,2}$, JIANG Hao ${ }^{1}$, CHEN Yong-fu' ${ }^{2}$, YIN Chang-bin ${ }^{1}$ \\ (1. Institute of Agricultural Resources and Regional Planning, Chinese Academy of Agricultural Sciences, \\ Beijing 100081, China; 2. College of Economics and Management, China Agricultural University, Beijing \\ 100083, China)
}

\begin{abstract}
In order to promote farmers' active participation in pollution control, based on the perspective of willingness-to-behavior transformation, we used the survey data of pig-scale farmers in 25 counties of Jilin and Liaoning provinces to group the sample data (no willingness or behavior, willingness without behavior, willingness to have behavior). And then, the influencing factors of pig manure disposal behavior in large- scale farming were comprehensively analyzed by using disordered multi-classification Logistic regression model from three dimensions of "willingness or no willingness", "behavior with or without" and "willingness to transform behavior". The results showed that there was a great difference between the willingness and behavior of manure management among farmers, and the inconsistency rate of willingness and behavior was $26.8 \%$. The factors influencing the farmers' willingness to transform the manure disposal behavior are mainly related to the scale of farming, the area of manure disposal area, the net income of farming, the surrounding public opinion, the technology of manure disposal, the economic conditions of manure disposal, the training of manure disposal, government subsidies and government supervision, and have a significant role in promoting the willingness to be transformed into behavior. However, the cultivation years have become an obstacle to the conversion of farmers' willingness to behavior transformation.
\end{abstract}

Keywords: scale pig breeding; manure pollution treatment; treatment behavior; consistency; influencing factor 